\title{
ESTRATÉGIAS DE AGRUPAMENTO DE DADOS PARA A MODELAGEM HIPSOMÉTRICA E SEUS REFLEXOS NA ESTIMATIVA DE VOLUME EM PLANTIOS DE Pinus spp.
}

\author{
Sintia Valerio Kohler1*, Afonso Figueiredo Filho ${ }^{2}$, Henrique Soares Koehler ${ }^{3}$, Julio Eduardo Arce ${ }^{4}$, \\ Fabiane Aparecida de Souza Retslaff ${ }^{5}$, Edson Luis Serpe ${ }^{5}$ \\ ${ }^{1 *}$ Universidade Federal do Paraná, Programa de Pós-Graduação em Engenharia Florestal, Curitiba, Paraná, Brasil - \\ sintiakohler@yahoo.com.br \\ ${ }^{2}$ Universidade Estadual do Centro-Oeste, Departamento de Engenharia Florestal, Irati, Paraná, Brasil - afigfilho@gmail.com; \\ faretslaff@gmail.com \\ ${ }^{3}$ Universidade Federal do Paraná, Departamento de Fitotecnia e Fitossanitarismo, Curitiba, Paraná, Brasil - koehler@ufpr.br \\ ${ }^{4}$ Universidade Federal do Paraná, Departamento de Ciências Florestais, Curitiba, Paraná, Brasil - jarce@ ufpr.br \\ ${ }^{5}$ Universidade Estadual do Centro-Oeste, Programa de Pós-Graduação em Ciências Florestais, Irati, Paraná, Brasil - serpe.edson @ gmail.com \\ Recebido para publicação: 07/02/2017 - Aceito para publicação: 23/09/2017
}

\begin{abstract}
Resumo
O volume das árvores é uma das variáveis de maior interesse no inventário florestal, cuja estimativa é feita com base no diâmetro e/ou altura das árvores. O diâmetro é facilmente obtido, ao passo que a medição das alturas é uma atividade onerosa que demanda tempo e altos custos. Assim, o uso de modelos hipsométricos é uma atividade frequente em inventários florestais. Este trabalho buscou avaliar estratégias para o ajuste desses modelos e o efeito das alturas estimadas na predição do volume do povoamento. Em uma área de 43,579 hectares de plantio misto de Pinus spp., foi realizado um censo com a medição dos diâmetros e das alturas de todas as árvores. Após o censo, foi realizado um inventário por amostragem, com instalação aleatória de 37 parcelas, e cubagem de 102 árvores. Foram testadas quatro estratégias de agrupamento de dados para ajuste de modelos hipsométricos utilizando dados de altura e de diâmetro obtidos das parcelas, da cubagem e das árvores dominantes. A utilização somente dos dados da cubagem pode ser suficiente para o ajuste de um modelo hipsométrico único, gerando boas estimativas de altura. Em inventários onde não há a necessidade de cubagem de árvores, um único ajuste pode ser suficiente, a partir da medição de cerca de 10 árvores por parcela, mais as alturas das árvores dominantes. A estratégia que produziu melhores estimativas aliada à menor necessidade de medições de altura em campo é a que utiliza dados das árvores cubadas em conjunto com as árvores dominantes, ajustando-se um único modelo.

Palavras-chave: Altura das árvores; estoque de madeira; censo.
\end{abstract}

\begin{abstract}
Data grouping strategies for height-diameter modeling and its reflections on volume estimation in Pinus spp. stand. Tree volume is one of the variable of greatest interest in a forest inventory and its estimate is based on the diameter and/or tree heights. Diameter is easily obtained, while measuring tree height is difficult activity that demands time and resources. Thus, fitting height-diameter models is a current activity in forest inventories. This work aimed to evaluate the strategies for fitting a height-diameter model, as well as the effect of estimated heights in the prediction of volumetric stock of forests stands. In an area of 43.579 hectares of Pinus spp mixed stand, a census was carried out, where the diameter and heights of all trees were measured. After the census, an inventory was carried out by sampling, setting 37 plots, distributed randomly in the stand, and also 102 trees were sampled for log scaling. Four data clustering approaches were tested to fit the height-diameter model, using data of height and diameter obtained from plots, tree scaling and dominant tree. Using only the data obtained in the tree scaling can be enough to fit a single height-diameter model, providing good estimates of tree height. In an inventory where tree scaling is not required, a single fit can be enough to represent the stand, measuring about 10 trees per plot, plus the heights of the dominant trees. The strategy that produced the best estimates combined with the minor need of height measurements is the one that uses data from tree scaling along with dominant tree heights, resulting in a single model.

Keywords: Trees height; wood stock; census.
\end{abstract}

\section{INTRODUÇÃO}

O Brasil possui extensas áreas de florestas plantadas, principalmente com as espécies dos gêneros Eucalyptus e Pinus, que constituem a base de importantes atividades industriais. A necessidade das empresas florestais em promover melhorias nas metodologias para quantificar e estimar a produção dessas florestas é

FLORESTA, Curitiba, PR, v. 47, n. 3, p. 307 - 316, jul. / set. 2017

Kohler, S. V. et al.

ISSN eletrônico 1982-4688

DOI: $10.5380 /$ rf.v47i1.50555 
constante. Segundo Silva et al. (2007), a estimativa de produção de madeira em florestas normalmente é feita por procedimentos de inventário florestal, e, para estimar o volume individual da árvore, busca-se relacionar variáveis como o diâmetro à altura do peito e a altura total da árvore com o seu volume.

O volume das árvores é uma das variáveis de maior interesse ao se realizar um inventário florestal, sua estimativa é geralmente obtida com modelos ajustados por regressão, com base, principalmente, nas variáveis diâmetro e altura. Em inventários florestais, a variável diâmetro à altura do peito é facilmente medida para todas as árvores, entretanto, a altura, quando mensurada com hipsômetros apropriados, fornece resultados acurados, porém não econômicos, devido ao tempo gasto em sua obtenção. Devido a isso, normalmente se medem as alturas de algumas árvores com hipsômetros e a partir da composição de uma amostra que contenha os diâmetros e alturas das árvores, ajustam-se relações hipsométricas que permitirão estimar as alturas das demais árvores (SILVA et al., 2012).

Segundo Machado et al. (1994), a relação hipsométrica não é uma relação dendrométrica muito forte, existindo uma grande variabilidade de alturas para uma mesma classe de diâmetro, resultando, na maioria dos casos, em estatísticas de ajuste e exatidão com valores aquém dos desejados em análise de regressão. Em idades mais avançadas e em povoamentos com estrutura alterada por desbastes, também não são muito bons os ajustes. As dificuldades em modelar relações hipsométricas está no fato de que curvas de altura/diâmetro são influenciadas por vários fatores como posição sociológica, qualidade de sítio, idade, densidade, tratamentos silviculturais e características da própria espécie (BARROS et al., 2002; MACHADO et al., 2011).

Outro aspecto a considerar no uso de relações hipsométricas é o conjunto de dados (amostra) usados para ajuste dos modelos. Existem várias alternativas atualmente empregadas como o ajuste de um modelo por parcela, ajuste com dados medidos de todas as parcelas, usando somente dados da cubagem de árvores, entre muitas outras possibilidades. A forma de abordagem do conjunto de dados certamente é um fator que pode influenciar nas estimativas finais do volume da população.

Muitos autores empregaram estratégias diferentes para compor a amostra nos ajustes de modelos hipsométricos. Como exemplo, Pelissari et al. (2016) que, após testarem diferentes conjuntos de diâmetros e alturas, sugeriam a medição de $40 \%$ das árvores em cada parcela em povoamentos de teca, Nicoletti et al. (2016) utilizaram a medição das 20 primeiras árvores de cada parcela, além das dominantes para ajustar modelos em diferentes fases do ciclo de corte em plantios de Pinus taeda L.; Oliveira et al. (2014) utilizaram a medição de altura das seis primeiras árvores de cada parcela um plantio de Eucalyptus sp.; Retslaff et al. (2015), para estimar alturas em diferentes idades de plantios de Eucalyptus grandis Hill ex Maiden, utilizaram a medição de alturas das 10 primeiras árvores mais as dominantes por parcela; Thiersch et al. (2013), para unidades amostrais circulares de $500 \mathrm{~m}^{2}$, mediram a altura total de 10 árvores centrais e de cinco dominantes em povoamentos clonais de Eucalyptus spp.; e ainda Machado et al. (1994) testaram vários modelos hipsométricos utilizando alturas medidas das primeiras 25 a 30 árvores por parcela, para ajustes por grupos de idades em povoamentos de Pinus elliottii Engelm.

Em várias pesquisas têm-se procurado determinar o melhor conjunto de dados e a melhor forma de ajustes de modelos hipsométricos, como no caso de Ribeiro et al. (2010), onde os autores testaram diferentes estratégias e metodologias de coleta de dados para ajustes de modelos hipsométricos tradicionais e genéricos para plantios de Eucalyptus spp. As metodologias de coleta de dados envolveram a medição das cinco primeiras árvores da parcela na primeira estratégia e a adição de árvores dominantes e árvores que não foram representadas em todos os quartis baseados nos diâmetros. Os ajustes ainda foram realizados por parcela, por idade, por região e por estrato (combinação da região com a idade). Silva et al. (2007) analisaram a influência de diferentes tamanhos e composições de amostras no ajuste de modelos hipsométricas para Eucalyptus grandis. Soares et al. (2004) testaram diferentes alternativas de conjunto de dados para ajustes de modelos hipsométricos, realizando ajustes por parcela, idade, sítios, combinação de idade e sítio e agrupando todas as parcelas.

Sabe-se que, para a correta aplicação da técnica de regressão, toda amplitude de variação dos dados deve ser medida ou captada no processo amostral. Sendo assim, a metodologia selecionada para escolher quais árvores da parcela deverão ser mensuradas as alturas terá influência no desempenho ou na qualidade preditiva dos modelos que descrevem a relação hipsométrica (RIBEIRO et al., 2010). Contudo, a maior parte dos estudos não avalia a influência da estratégia empregada para estimativa de alturas sobre o estoque volumétrico do povoamento. Dessa forma, esta pesquisa buscou avaliar estratégias de ajustes de modelos hipsométricos e seus efeitos na estimativa do estoque volumétrico, comparando-o com o valor paramétrico obtido pelo censo de um povoamento de Pinus spp. Buscou-se, portanto, testar a hipótese de que a estratégia de agrupamento de dados para ajuste do modelo hipsométrico tem influência na estimativa do estoque volumétrico do povoamento. 


\section{MATERIAL E MÉTODOS}

\section{Caracterização da base de dados}

Foram coletados dados de povoamentos de Pinus spp. pertencentes a empresa REMASA Reflorestadora S.A., com sede no município de Bituruna, região Sudeste do estado do Paraná. A altitude varia entre 900 a $1.100 \mathrm{~m}$, com topografia fortemente ondulada a montanhosa. Os solos da região apresentam textura argilosa e forte declividade. O clima, segundo a classificação de Köppen, é o subtropical úmido mesotérmico (Cfb) com temperatura média anual de $16^{\circ} \mathrm{C}$, sem estação seca definida, com verões frescos e geadas severas e frequentes no inverno, com chuvas variando entre 1.600 e 1.900 mm (MAACK, 2012; ALVARES et al., 2013).

A área total do povoamento é de 43,579 hectares em um plantio misto com Pinus taeda L. e Pinus elliottii Engelm. de 16 anos de idade. Inicialmente, foram plantadas 1.111 árvores por hectare com espaçamento $3 \mathrm{~m}$ x 3 m e realizados desbastes aos 9 e aos 12 anos, restando em média 444 árvores por hectare. A capacidade produtiva do povoamento é representada por sítios medianos e pobres, em que, segundo a classificação de sítio realizada com dados da região, a área possui sítios de qualidade III ( $24 \mathrm{~m})$ e IV $(21 \mathrm{~m})$.

Os parâmetros da população foram obtidos por meio do censo de toda a área, sendo medidos o diâmetro a $1,3 \mathrm{~m}$ do solo $(d)$ e a altura total $(h)$ de todas as árvores presentes no povoamento, totalizando 19.329 árvores medidas. Adicionalmente, foi realizado um inventário por amostragem com intensidade amostral de 5\%, totalizando 37 unidades amostrais circulares com $600 \mathrm{~m}^{2}$ distribuídas aleatoriamente (Figura 1). Em cada parcela foram medidos os diâmetros $(d)$ de todas as árvores e a altura total das 10 primeiras árvores, mais a altura das árvores dominantes pelo critério de Assmann, selecionando-se seis árvores dominantes por parcela.

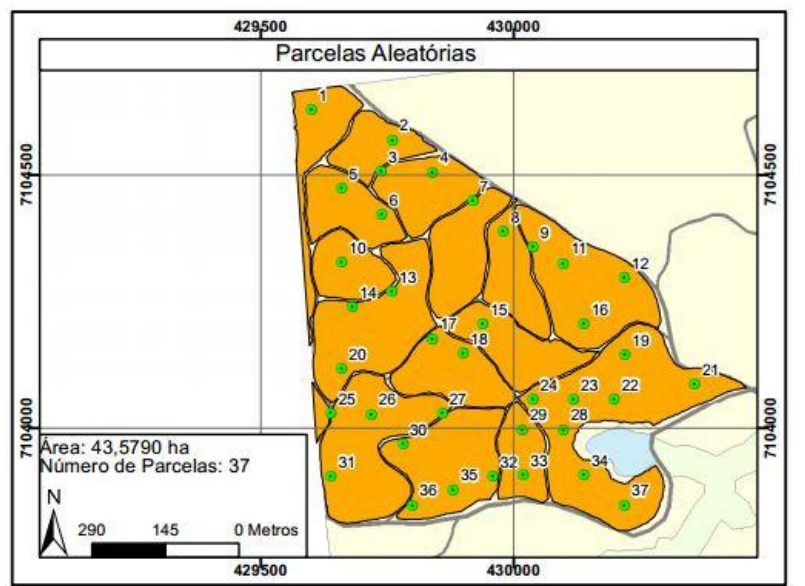

Figura 1. Distribuição aleatória das parcelas amostradas no povoamento de Pinus spp.

Figure 1. Random distribution of sampled plots in the Pinus spp. stand.

Foram cubadas 102 árvores selecionadas abrangendo todas as classes de diâmetro presentes na população. As árvores foram cubadas utilizando a fórmula de Smalian, medindo-se os diâmetros nas alturas absolutas $(0,1$ e $1,30 \mathrm{~m})$ e relativas $(10,15,25, \ldots, 95 \%$ da altura total $)$.

\section{Alturas estimadas das árvores}

Uma prática comum em inventários florestais é a medição de apenas parte das alturas das árvores das unidades amostrais, com a qual ajustam-se modelos hipsométricos para estimar as demais alturas. Neste trabalho, os ajustes foram efetivados com dados de $d$ e $h$ de diferentes conjuntos de dados. Cada grupo representou um tratamento, conforme descrito a seguir:

- Tratamento 0: Alturas de todas as árvores das parcelas obtidas com o censo (testemunha).

- Tratamento 1: Ajuste do modelo com os dados de todas as parcelas, sendo medida a altura das 10 primeiras árvores mais 6 árvores dominantes de cada parcela.

- Tratamento 2: Ajuste do modelo, utilizando apenas $d$ e $h$ das árvores cubadas.

- Tratamento 3: Ajuste do modelo com dados das árvores medidas nas parcelas (10 primeiras árvores mais 6 árvores dominantes de cada parcela) junto os dados das árvores cubadas.

- Tratamento 4: Ajuste do modelo apenas com dados das árvores dominantes das parcelas mais as árvores cubadas. 
Inicialmente foram testados vários modelos hipsométricos com os dados coletados. Essas análises preliminares apontaram o modelo (1) de Stoffels e Van Soest (1953) como o mais adequado às várias modalidades de conjuntos de dados, sendo então, selecionado para os demais ajustes.

$$
\ln h=\beta_{0}+\beta_{1} \ln d+\varepsilon_{i}
$$

em que: $d=$ diâmetro a $1,30 \mathrm{~m}$ do solo $(\mathrm{cm}) ; h=$ altura total da árvore $(\mathrm{m}) ; \mathrm{ln}=$ logaritmo neperiano; $\beta_{i}=$ coeficientes do modelo; $\varepsilon_{i}=$ erro aleatório.

O modelo de Stoffels e Van Soest foi ajustado para cada tratamento e seu desempenho foi avaliado com base no coeficiente de determinação $\left(\mathrm{R}^{2}\right)$, erro padrão da estimativa recalculado para altura total $\left(\mathrm{S}_{\mathrm{yx}} \%\right)$ e análise gráfica dos resíduos. Os coeficientes do modelo, obtidos com os dados de cada tratamento, foram utilizados para estimar a altura das demais árvores que não tiveram suas alturas reais medidas em campo no momento do inventário. Assim, foram gerados quatro grupos diferentes de alturas estimadas para as mesmas árvores, além de suas alturas reais, obtidas com o censo (testemunha).

Para comparar os diferentes grupos de alturas estimadas foi realizada uma análise de variância utilizado o delineamento inteiramente casualizado. A hipótese de nulidade testada foi: $\mathrm{H}_{0}: \mu_{1}=\mu_{2} \ldots=\mu_{n}$; sendo $\mu_{1}, \mu_{2} \ldots$ $\mu_{n}$, as alturas médias das parcelas dos tratamentos 1 a 5 (n), em que cada parcela representou uma repetição do tratamento, totalizando 37 repetições. Anteriormente a análise de variância, o teste de Bartlett foi aplicado para verificar a homogeneidade das variâncias dos tratamentos. O nível de 5\% de significância foi utilizado para essas análises.

\section{Volume estimado das árvores}

Para estimativa do volume total individual das árvores medidas na amostragem, foi ajustado o modelo (2) de Schumacher e Hall (1933). Visando a obtenção de estimativas mais exatas dos volumes, devido à grande amplitude de diâmetros do povoamento, o modelo foi ajustado para classes de diâmetro, sendo elas: Árvores com diâmetros entre 12 e 26,9 cm (Classe I); diâmetros entre 27 e 41,9 cm (Classe II) e diâmetros entre 42 e $57 \mathrm{~cm}$ (Classe III).

$$
\ln v=\beta_{0}+\beta_{1} \ln d+\beta_{2} \ln h+\varepsilon_{i}
$$

em que: $v=$ volume total $\left(\mathrm{m}^{3}\right) ; d=$ diâmetro a $1,30 \mathrm{~m}$ do solo, $d(\mathrm{~cm}) ; h=$ altura total $(\mathrm{m}) ; \ln =\operatorname{logaritmo}$ neperiano; $\beta_{i}=$ coeficientes do modelo; $\varepsilon_{i}=$ erro aleatório

\section{Estimativa do estoque volumétrico do povoamento}

As alturas das árvores estimadas com as equações hipsométricas e as medidas em campo, com seus respectivos diâmetros, foram inseridos na equação (2), estimando-se os volumes individuais das árvores das parcelas. Aplicando os procedimentos de cálculo da amostragem aleatória, foi obtido o estoque total de madeira do povoamento. Foram obtidas cinco estimativas de volume para o povoamento, conforme o tratamento empregado na estimativa das alturas das árvores.

Para avaliar a acuracidade das estimativas do inventário, os valores de volume total por hectare estimados foram comparados com o valor paramétrico (volume total por hectare real), determinado pelo censo do povoamento. A diferença entre o valor paramétrico do volume e a estimativa do parâmetro possibilitou a determinação do erro real, obtido com a seguinte fórmula (3):

$$
\operatorname{Erro}(\%)=\frac{\left(V_{R}-V_{E}\right)}{V_{R}} .100
$$

em que: Erro $(\%)=$ erro real relativo $(\%) ; \mathrm{V}_{\mathrm{R}}=$ volume real obtido no censo florestal; $\mathrm{V}_{\mathrm{E}}=$ volume estimado obtido com o inventário por amostragem.

\section{RESULTADOS}

\section{Ajustes dos modelos hipsométricos}

As estatísticas e coeficientes do modelo hipsométrico ajustado com os dados dos quatro tratamentos, estão apresentadas na tabela 1 . O Tratamento 0 (testemunha) é caracterizado pela medição da altura de todas as árvores das parcelas, dessa forma não demandou o ajuste de modelos hipsométricos. 
Os melhores resultados nos ajustes foram obtidos com os Tratamentos 2 e 4, embora tenham sido ajustados com um número de observações menor (n). No Tratamento 2, apenas as alturas das árvores cubadas são empregadas no ajuste, o coeficiente de determinação $\left(\mathrm{R}^{2}\right)$ encontrado foi de 0,57 e o erro padrão de estimativa $\left(\mathrm{S}_{\mathrm{yx}}\right) 7,3 \%$. No Tratamento 4 , além das alturas das árvores cubadas, foram inseridos no ajuste, as alturas das árvores dominantes das parcelas, resultando em um coeficiente de determinação menor que a opção anterior $(0,34)$, porém, com um menor erro padrão de estimativa $(6,7 \%)$.

Tabela 1. Estatísticas e coeficientes do modelo hipsométrico ajustado para cada tratamento com dados de um povoamento de Pinus spp.

Table 1. Statistics and coefficients of hypsometric model fitted for each treatment with data of a Pinus spp. stand.

\begin{tabular}{lcccccc}
\hline \multirow{2}{*}{ Tratamento } & \multirow{2}{*}{$\mathbf{N}^{\circ}$ observações (n) } & \multirow{2}{*}{$\mathbf{R}^{\mathbf{2}}$} & $\mathbf{S}_{\mathbf{y x}}(\mathbf{m})$ & $\mathbf{S}_{\mathbf{y x}} \mathbf{\%}$ & \multicolumn{2}{c}{ Coeficientes } \\
\cline { 6 - 7 } & 590 & 0,21 & 1,47 & 7,8 & $2,1411^{* *}$ & $0,2319^{* * *}$ \\
T1 & 102 & 0,57 & 1,41 & 7,3 & $2,0795^{* *}$ & $0,2539 * *$ \\
T2 & 692 & 0,29 & 1,46 & 7,7 & $2,1094 * *$ & $0,2418^{* *}$ \\
T4 & 324 & 0,34 & 1,27 & 6,7 & $2,1287 * *$ & $0,2332^{* * *}$ \\
\hline
\end{tabular}

$\mathrm{T} 1=10$ primeiras árvores mais 6 árvores dominantes de cada parcela; T2 = árvores cubadas; T3 = 10 primeiras árvores e 6 árvores dominantes de cada parcela mais as árvores cubadas; $\mathrm{T} 4=6$ árvores dominantes de cada parcela mais as árvores cubadas; $\mathrm{R}^{2}=$ coeficiente de determinação; $S_{\mathrm{yx}}(\mathrm{m})=$ erro padrão da estimativa absoluto; $\mathrm{S}_{\mathrm{yx}} \%=$ erro padrão da estimativa relativo; $\mathrm{b}_{0}$ e $\mathrm{b}_{1}=$ coeficiente das equações; $* *$ $=$ coeficiente significativo ao nível de $5 \%$ de probabilidade.

Constata-se que a dispersão dos resíduos para os tratamentos não apresentou tendências (Figura 2). As linhas dos valores estimados possuem uma inclinação muito suave, aproximando-se de uma reta paralela ao eixo X. Os resultados apresentados na figura 2 indicam que os modelos ajustados produziram boas estimativas para a altura total em toda a extensão dos dados, ao passo que a linha de estimativa acompanhou a dispersão dos pontos representada pelos valores observados das alturas, explicando o baixo erro padrão de estimativa gerado em todos os ajustes.

Observa-se que a dispersão dos resíduos para os tratamentos não apresentou tendências (Figura 2). As linhas dos valores estimados possuem uma inclinação muito suave, aproximando-se de uma linha reta paralela ao eixo $\mathrm{X}$, ou seja, para qualquer valor de $\mathrm{X}$, o modelo estima alturas das árvores próximo à média (valor do intercepto $\beta_{0}$ ), explicando os baixos coeficientes de determinação obtidos. Esse fato é comumente observado quando se analisa povoamentos em que o crescimento em altura está estabilizado devido as idades mais avançadas, desbastes ou baixa capacidade de sítio, podendo chegar ao ponto de não existir regressão para a relação h/d.
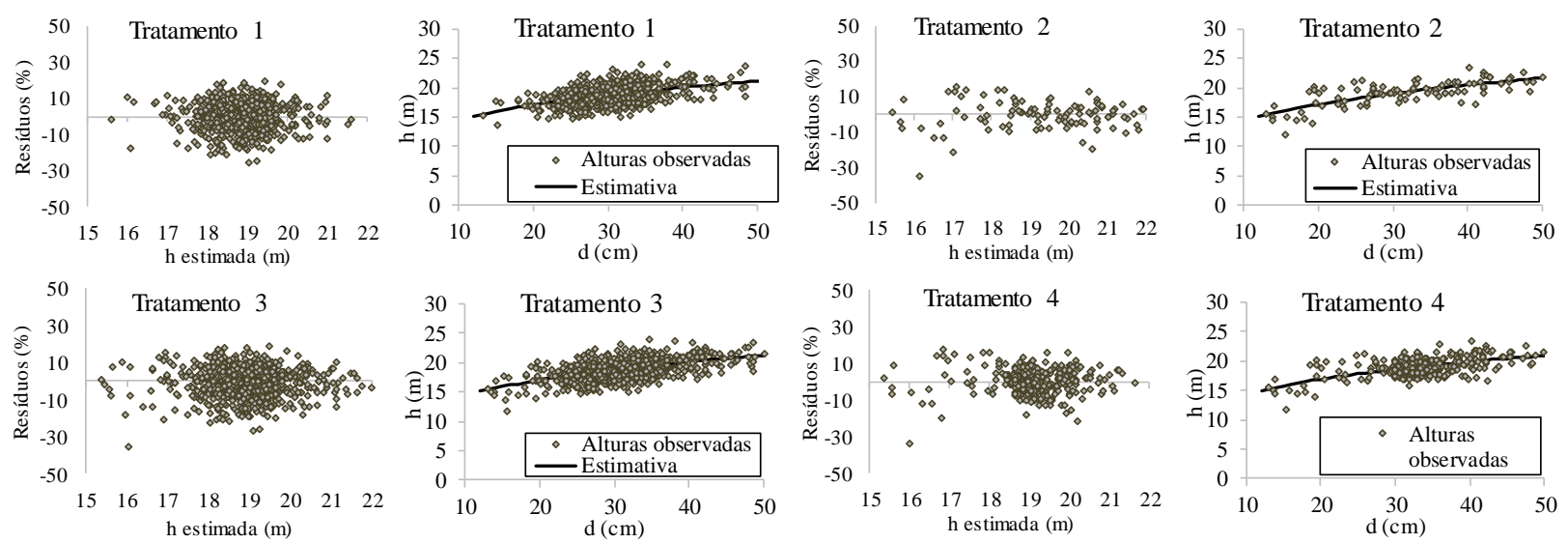

Figura. 2. Dispersão dos resíduos e dos valores observados sobre a curva ajustadas para os tratamentos 1, 2, 3 e 4 em um povoamento de Pinus spp.

Figure. 2. Dispersion of the residues and observed values on the fitted curve for treatments $1,2,3$ and 4 in a Pinus spp. stand.

\section{Comparação das alturas estimadas entre os tratamentos}

As estatísticas das alturas estimadas com as equações hipsométricas, obtidas de acordo com cada tratamento, estão apresentadas na tabela 2. O coeficiente de variação do Tratamento 0, onde todas as árvores tiveram sua altura medida em campo, é maior do que os demais tratamentos. Em geral, os tratamentos apresentam valores estimados semelhantes, com uma altura média estimada muito próxima. O coeficiente de

FLORESTA, Curitiba, PR, v. 47, n. 3, p. 307 - 316, jul. / set. 2017

Kohler, S. V. et al.

ISSN eletrônico 1982-4688

DOI: $10.5380 /$ rf.v47i1.50555 
Pearson, indica que a correlação entre a altura e o diâmetro das árvores do povoamento é moderada, estando entre 0,45 a 0,55 .

Tabela 2. Estatísticas das alturas totais estimadas com diferentes conjuntos dos dados para um povoamento de Pinus spp.

Table 2. Statistics of total heights estimated with different data grouping strategies for a Pinus spp. stand.

\begin{tabular}{lccccc}
\hline \multirow{2}{*}{ Estatística } & \multicolumn{5}{c}{ Tratamentos } \\
\cline { 2 - 5 } & T0 & T1 & T2 & T3 & T4 \\
\hline Média $(\mathrm{m})$ & 18,63 & 18,65 & 18,73 & 18,66 & 18,66 \\
Variância $\left(\mathrm{m}^{2}\right)$ & 2,92 & 1,88 & 1,88 & 1,89 & 1,89 \\
Desvio padrão & 1,71 & 1,37 & 1,37 & 1,37 & 1,37 \\
CV \% & 9,18 & 7,35 & 7,31 & 7,37 & 7,36 \\
$\mathrm{r}$ & 0,46 & 0,55 & 0,55 & 0,56 & 0,56 \\
\hline
\end{tabular}

T0 = Todas as alturas medidas nas parcelas; T1 = 10 primeiras árvores mais 6 árvores dominantes de cada parcela; T2 = árvores cubadas; T3 = 10 primeiras árvores e 6 árvores dominantes de cada parcela mais as alturas das árvores cubadas; T4 = árvores dominantes das parcelas e árvores cubadas; $\mathrm{CV} \%$ = coeficiente de variação; $r=$ coeficiente de correlação de Pearson para diâmetro e altura.

O teste de Bartlett mostrou que os dados possuem variâncias homogêneas. Dessa forma, seguiu-se com a análise de variância, que apresentou um p-valor de 0,994 para o teste $\mathrm{F}$, indicando que não existem diferenças entre as alturas estimadas, ao nível de 5\% de significância. Assim, os conjuntos de dados usados para estimar as alturas não diferem entre si e também não diferem da média real das alturas, representada pelo Tratamento 0 .

\section{Efeito da estratégia de ajuste da relação hipsométrica nas estimativas de volume do povoamento}

As estatísticas de ajuste e precisão (Tabela 3) indicaram que o modelo de Schumacher e Hall foi adequado aos dados, apresentando erro padrão de estimativa de no máximo 10,5\% e coeficiente de determinação superiores a 0,70. Os ajustes foram melhores para as classes de diâmetros inferiores, ao passo que a distribuição de resíduos foi adequada em todas as classes (Figura 3), não apresentando tendências relevantes nas estimativas dos volumes das árvores.

Tabela 3. Estatísticas obtidas com o ajuste do modelo de volume para as classes de diâmetro em um povoamento de Pinus spp.

Table 3. Statistics obtained with the fit of volume model for diameter classes in a Pinus spp. stand.

\begin{tabular}{|c|c|c|c|c|c|c|c|}
\hline \multirow{2}{*}{ Classes de $d$} & \multirow{2}{*}{$\begin{array}{c}\mathbf{N}^{\circ} \text { obs. } \\
\text { (n) }\end{array}$} & \multirow{2}{*}{$\mathbf{R}^{2}$} & \multirow{2}{*}{$\begin{array}{c}\mathbf{S}_{\mathbf{y x}} \\
\left(\mathbf{m}^{3}\right)\end{array}$} & \multirow{2}{*}{$\begin{array}{l}S_{\mathrm{yx}} \\
(\%)\end{array}$} & \multicolumn{3}{|c|}{ Coeficientes } \\
\hline & & & & & $b_{0}$ & $\mathbf{b}_{1}$ & $\mathbf{b}_{2}$ \\
\hline I & 31 & 0,98 & 0,022 & 7,1 & $-10,5964 * *$ & $1,8503^{* *}$ & $1,3173 * *$ \\
\hline II & 46 & 0,88 & 0,079 & 9,1 & $-9,1611^{* *}$ & $1,7303 * *$ & $0,9717 * *$ \\
\hline III & 25 & 0,75 & 0,159 & 10,5 & $-8,5375^{* *}$ & $1,5238 * *$ & $1,0115^{* *}$ \\
\hline
\end{tabular}

$\mathrm{R}^{2}$ = coeficiente de determinação; $S_{y x}=$ erro padrão da estimativa absoluto; $S_{y x} \%=$ erro padrão da estimativa relativo; $b_{0}, b_{1}$ e $b_{1}=$ coeficiente das equações; $* *=$ coeficiente significativo ao nível de $5 \%$ de probabilidade.

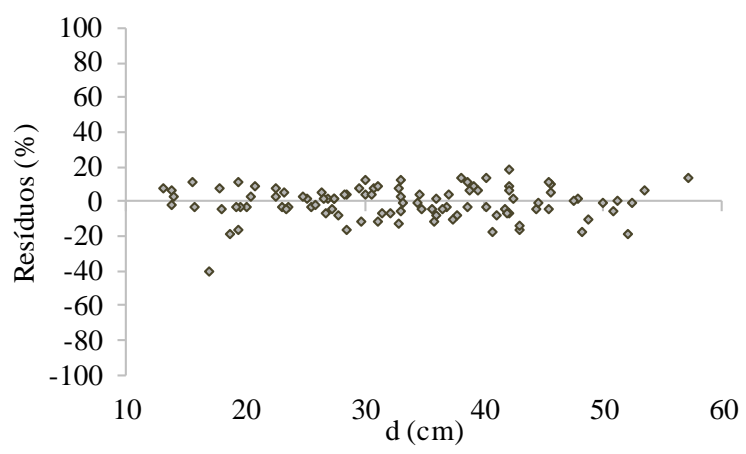

Figura 3. Distribuição dos resíduos para volumes individuais estimados com o modelo de Schumacher e Hall ajustado em classe diamétrica em um povoamento de Pinus spp.

Figure 3. Distribution of residuals to individual volumes estimated with the Schumacher and Hall's model adjusted in diametric class in a Pinus spp. stand. 
As equações volumétricas geradas para cada classe de diâmetro foram empregadas para obter o valor paramétrico do volume da população, sendo determinado o volume individual de cada uma das 19.329 árvores com diâmetros $(d)$ e alturas mensuradas no censo. As mesmas equações também foram usadas para estimar o volume das árvores das parcelas medidas na amostragem e submetidas aos diferentes tratamentos para estimativa das alturas.

De acordo com o censo, existiam em média 444 árvores por hectare no povoamento, gerando um volume total de $277,1 \mathrm{~m}^{3}$ por hectare e $12.070,6 \mathrm{~m}^{3}$ na área total de 43,579 hectares. Os volumes do povoamento estimados pelo inventário florestal por amostragem estão apresentados na figura 4, onde foram obtidos cinco valores diferentes, de acordo com os tratamentos empregados na estimativa das alturas. Assim, observou-se que em todos os tratamentos houve superestimativa do volume em relação ao valor paramétrico. O Tratamento 3, que apresentou as melhores estatísticas de ajuste, resultou na estimativa de volume mais distante do valor paramétrico $\left(291,5 \mathrm{~m}^{3} \mathrm{ha}^{-1}\right)$. Os demais tratamentos estimaram volumes semelhantes, em torno de $287 \mathrm{~m}^{3}$ por hectare.

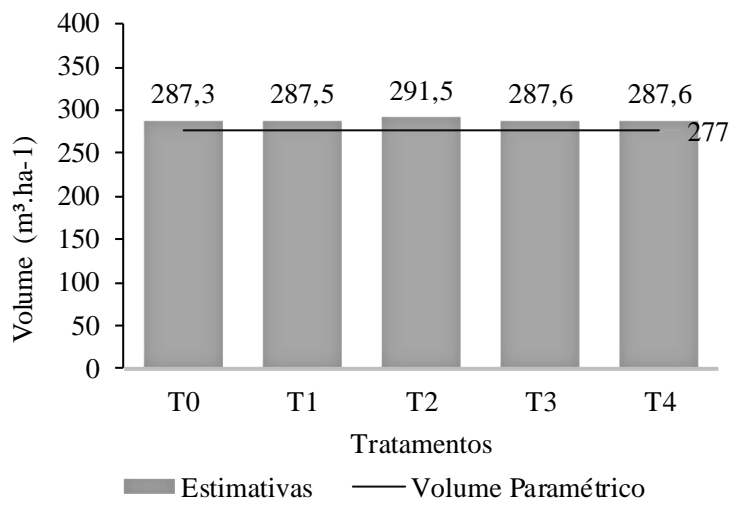

Figura 4. Volume por hectare estimados de acordo com os tratamentos para estimativa de alturas em um povoamento de Pinus spp.

Figure 4. Volume per hectare estimated according to the treatments for height estimation in a Pinus spp. stand.

O erro de amostragem (Tabela 4) foi baixo para todos os tratamentos (5,8\% em média). O erro real (Erro \%), obtido pela diferença entre os volumes estimados e o volume paramétrico foi baixo para todos os tratamentos, indicando que o volume estimado para o povoamento foi próximo ao real (volume paramétrico). Observou-se também que os valores negativos do erro real confirmaram a tendência observada anteriormente de superestimava dos volumes.

Tabela 4. Erro real e erro amostral para o volume estimado pelo inventário em cada tratamento em um povoamento de Pinus spp.

Table 4. Real and sample errors for the volume estimated by inventory in each treatment in a Pinus spp. stand.

\begin{tabular}{lcc}
\hline Tratamentos & Erro de amostragem $(\%)$ & Erro $\% *$ \\
\hline T0 & 6,2 & $-3,7$ \\
T1 & 5,9 & $-3,7$ \\
T2 & 6,0 & $-5,2$ \\
T3 & 5,9 & $-3,8$ \\
T4 & 5,9 & $-3,8$ \\
\hline ela diferença entre o volume por hectare estimado e o volume por hectare real obtido com o censo.
\end{tabular}

* = erro real obtido pela diferença entre o volume por hectare estimado e o volume por hectare real obtido com o censo.

T0 = Medição da altura de todas as árvores (testemunha); T1 = 10 primeiras árvores mais 6 árvores dominantes de cada parcela; T2 = árvores cubadas; T3 = 10 primeiras árvores e 6 árvores dominantes de cada parcela mais as árvores cubadas; T4 = árvores dominantes das parcelas e árvores cubadas.

\section{DISCUSSÃO}

\section{Análise dos conjuntos de dados usados para estimativas de alturas}

Os tratamentos que utilizaram árvores cubadas (T2) e a combinação de árvores cubadas e dominantes por parcela (T4) foram as duas melhores opções para ajuste do modelo hipsométrico, levando em consideração as estatísticas encontradas. Todos os tratamentos apresentaram características de ajuste do modelo hipsométrico 
semelhantes, as linhas dos valores estimados apresentaram inclinação muito suave, aproximando-se de uma linha reta paralela ao eixo $\mathrm{X}$.

Sabe-se que curvas da relação hipsométrica são dinâmicas, ou seja, se deslocam para a direita, sobem de patamar e tornam-se menos inclinadas com o avanço da idade, como demonstrado por Figueiredo Filho et al. (2010) para Araucaria angustifolia (Bert.) O. Ktze. O povoamento em questão, apesar de não apresentar uma idade avançada (16 anos), possuía essa característica, cujas curvas mostraram-se bastante suavizadas e próximas a se tornarem paralelas ao eixo X. Em decorrência disso, a relação entre a altura e o diâmetro não é forte, como demostrado pelo coeficiente de correlação de Pearson (em torno de 0,5). Isso explica também o baixo coeficiente de determinação encontrado para os ajustes.

A variação no diâmetro altera apenas levemente a altura estimada, resultado que certamente está relacionado à estabilização do crescimento em altura do povoamento, além do mesmo ter sofrido dois desbastes ao longo de seu desenvolvimento. Esses eventos têm como consequência a descaracterização da relação altura/diâmetro. A mesma tendência foi observada por Barros et al. (2002) ao ajustar modelos hipsométricos para povoamentos de Pinus oocarpa Schiede sob diferentes tratamentos (idades e número de desbastes). Os autores constataram que coeficientes de determinação e erros padrão de estimativa baixos ocorrem devido a maior perda de indivíduos por desbastes seletivos. Essa tendência também é observada por Machado et al. (2011), ao constatarem que a medida que se aumentou o número de desbastes, os coeficientes de determinação tornaram-se mais baixos, porém o erro padrão de estimativa se manteve inferior a $10 \%$.

Com a realização dos desbastes, as árvores menores são removidas, ocasionando a uniformização da altura do povoamento. O maior espaçamento faz com que as árvores tenham maior crescimento em diâmetro e, dessa forma, para uma grande variação de diâmetros, tem-se pouca variação de altura. Machado et al. (2008), ao ajustarem modelos hipsométricos para um povoamento de Araucaria angustifolia, encontraram curvas de relação hipsométrica pouco íngremes. Os autores comentam que esse é um resultado esperado para povoamentos com idades mais avançadas ou estabelecidos em sítios de qualidade média ou baixa, como no povoamento do presente estudo.

Os conjuntos de dados usados para estimar as alturas não diferiram entre si e da média real das alturas, segundo a análise de variância aplicada às alturas estimadas. Não havendo diferenças estatísticas entre os conjuntos de dados, poder-se-ia optar pelo método mais simples e com menos medições de alturas (T2), resultando em redução de custos e tempo na realização do inventário. Os dados de diâmetros e alturas obtidos com a cubagem de árvores nos inventários fornecem informações suficientes para a estimativa das alturas das árvores das parcelas. Na cubagem a altura da árvore é obtida diretamente sobre o tronco, eliminando a necessidade de equipamentos especializados e diminuindo possíveis fontes de erros de medição. No entanto, esta alternativa tem validade somente se houver necessidade da cubagem de árvores, se já existirem equações volumétricas e de afilamento disponíveis para o povoamento, não é necessário a cubagem de árvores. Se não forem cubadas árvores no inventário, a alternativa mais adequada seria medir parte das árvores das parcelas e ajustar um modelo hipsométrico com todos os dados (T1), alternativa comumente empregada nos inventários de florestas plantadas no Brasil.

Andrade e Leite (2011) avaliaram o ajuste de modelos hipsométricos utilizando dados de diâmetros e alturas medidas nas parcelas do inventário e na cubagem de árvores. Os autores constataram que existe maior acuracidade nas estimativas ao utilizar dados de altura e diâmetros medidos em 10 árvores das parcelas agrupados aos dados medidos na cubagem. Esses resultados estão de acordo com os obtidos nesta pesquisa, ou seja, utilizar as alturas das árvores cubadas nos ajustes da relação hipsométrica pode contribuir de forma relevante para a estimativa das alturas das árvores das parcelas.

Entretanto, deve-se considerar que a não constatação de diferença entres as estratégias de ajuste do modelo hipsométrico, podem estar relacionadas às características do povoamento analisado. Povoamentos que apresentem curva h/d mais inclinadas, certamente podem refletir melhor as diferenças entre os tratamentos empregados. Dessa maneira, mais estudos devem ser conduzidos para diferentes situações de povoamentos florestais e notadamente em idades mais jovens, principalmente, nas faixas de idades para desbastes.

\section{Estimativas de volume do povoamento}

As estimativas de volume médio por hectares, obtidas de acordo com as estratégias de estimativa de altura apresentaram valores maiores que o real, determinado pelo censo. Essa superestimava dos volumes, em parte, está associada ao desenho de amostragem que superestimou também o número de árvores por hectare do povoamento. O inventário por amostragem empregado estima 448 árvores por hectare, sendo, portanto, um acréscimo de quatro árvores em relação ao valor paramétrico.

O erro de amostragem encontrado para os tratamentos está no limite aceitável recomendado pela literatura (10\%). Esse erro depende da variabilidade da amostra, quando foram medidas todas as alturas das 
parcelas (Tratamento 0) obteve-se maiores coeficiente de variação e maior erro de amostragem. Nos demais tratamentos, as alturas não medidas foram estimadas pelas equações hipsométricas, sendo mais homogêneas que as medidas em campo e se aproximando da altura média do povoamento. Consequentemente, tem-se menor variabilidade dos dados, influenciando no erro de amostragem.

O erro real (Erro \%) encontrado indicou que o volume estimado para o povoamento foi próximo ao real. Observou-se também que os valores negativos do erro real confirmam a tendência observada anteriormente de superestimava dos volumes. Com os resultados obtidos na pesquisa, qualquer um dos conjuntos de dados usados para desenvolver equações hipsométricas poderia ser adotada em um inventário florestal com qualidade similar na estimativa dos volumes. Ressalte-se novamente que a idade e as características do povoamento provavelmente contribuíram para a igualdade das estimativas entre os tratamentos, em que o crescimento em altura está praticamente estabilizado. A igualdade entre os tratamentos foi confirmada também com a análise de variância aplicada aos volumes estimados por parcela, onde também não se constatou diferenças estatisticamente significativas entre os tratamentos.

Como os tratamentos apresentam volumes estimados semelhantes, pode-se optar por aquele que proporcionará menores custos para a empresa. O Tratamento 2 (T2) é o que demanda menor esforço nas medições de altura, onde foram usadas somente as alturas das árvores cubadas. Considerando que a prática de cubagem de árvores para ajustes de modelos de volume é normalmente realizada nos inventários, esse tratamento pode proporcionar redução considerável no tempo de medição e nos custos finais do inventário.

Observou-se, no entanto, que o valor do erro real para o Tratamento 2 é levemente superior aos demais (5,2\%). Apesar da ausência de diferença estatística entre as estimativas dos tratamentos, do ponto de vista econômico, a diferença absoluta entre o volume estimado e o paramétrico pode ser relevante para uma empresa florestal.

O Tratamento 4 apresentou superestimativa do volume total de madeira por hectare $\left(14,5 \mathrm{~m}^{3}\right)$, em relação ao valor paramétrico do povoamento. Em média, 55\% do volume desse povoamento pode ser destinado à serraria (toras com diâmetros acima de $23 \mathrm{~cm}$ ), cujo valor de mercado está em torno de $\mathrm{R} \$ 130,00$ por $\mathrm{m}^{3}$ (valor referente ao ano de 2014). Com base nessas informações, estimou-se que a diferença entre o real e a estimativa representou $\mathrm{R} \$ 1.038,50$ a mais por hectare e $\mathrm{R} \$ 45.256,5$ para todo o povoamento. A subestimativa ou superestimativa do volume por hectare no inventário florestal pode representar uma significativa diferença em termos econômicos, principalmente em povoamentos de grandes extensões.

Considerando essa análise econômica simples, a utilização de apenas a altura das árvores da cubagem pode resultar em diferenças relevantes em termos econômicos. Uma alternativa a esse tratamento (T2) seria a utilização do Tratamento 4 (T4), onde se adiciona as alturas de seis árvores dominantes de cada parcela à base de dados da cubagem. Esse tratamento proporcionou bom ajuste dos modelos hipsométricos e estimativas de volume próximas ao volume paramétrico, sendo que, a medição de árvores dominantes também é uma prática comum e muitas vezes obrigatória em inventários florestais para a definição do índice de sítio.

Realizando a mesma análise econômica para o Tratamento 4 tem-se que a superestimativa no volume total foi de $10,64 \mathrm{~m}^{3}$ por hectare, resultado em um valor de $\mathrm{R} \$ 761,0$ a mais por hectare e $\mathrm{R} \$ 33.163,3$ ao total do povoamento. Essa discrepância de apenas 1,4\% no erro real resultou em uma diferença de aproximadamente 15 mil reais ao final da colheita e comercialização da madeira do povoamento.

\section{CONCLUSÃO}

- A cubagem de uma amostra representativa de toda a variação de diâmetros e alturas do povoamento é suficiente para realizar o ajuste de um modelo hipsométrico único visando a estimativa de altura das árvores do inventário.

- Caso a cubagem não seja necessária nas atividades do inventário, um único ajuste para representar o povoamento (ou talhão), a partir da medição de cerca de 10 árvores por parcela, mais as alturas das árvores dominantes, é a estratégia mais adequada, a qual, em geral, tem sido adotada nos inventários florestais atuais.

- O conjunto de dados que produz melhores resultados com o menor esforço de medições de altura em campo é o que utiliza dados das árvores cubadas, juntamente com as alturas de árvores dominantes, ajustando-se uma equação geral para o povoamento.

\section{REFERÊNCIAS}

ALVARES, C. A.; STAPE, J. L.; SENTELHAS, P. C.; GONÇALVES, J. L. M.; SPAROVEK, G. Köppen's climate classification map for Brazil. Meteorologische Zeitschrift, Berlin, v. 22, n. 6, p. 711-728, 2013.

FLORESTA, Curitiba, PR, v. 47, n. 3, p. 307 - 316, jul. / set. 2017

Kohler, S. V. et al.

ISSN eletrônico 1982-4688

DOI: $10.5380 /$ rf.v47i1.50555 
ANDRADE, V. C. L.; LEITE, H. G. Hipsometric relationship modeling using data sampled in tree scaling and inventory plots. Árvore, Viçosa, v. 35, n. 1, p. 157-164, 2011.

BARROS, D. A.; MACHADO, S. A.; ACERBI JÚNIOR, F. W.; SCOLFORO, J. R. S. Comportamento de modelos hipsométricos tradicionais e genéricos para plantações de Pinus oocarpa em diferentes tratamentos. Boletim de Pesquisa Florestal, Colombo, n. 45, p. 3-28, 2002.

FIGUEIREDO FILHO, A.; DIAS, A. N.; KOHLER, S. V.; VERUSSA, A. A.; CHIQUETTO, A. L. Evolution of the hipsometric relationship in Araucaria angustifolia plantations in the mid-south region of Paraná state. Cerne, Lavras, v. 16, n. 3, p. 347-357, 2010.

MAACK, R.; Geografia física do Estado do Paraná. Ponta Grossa: UEPG, 4 ed. 2012, 526p.

MACHADO, S. A.; NASCIMENTO, R. G. M.; AUGUSTYNCZIK, A. L. D.; SILVA, L. C. R.; FIGURA, M. A.; PEREIRA, E. M.; TÉO, S. J. Comportamento da relação hipsométrica de Araucaria angustifolia no capão da Engenharia Florestal da UFPR. Pesquisa Florestal Brasileira, Colombo, n. 56, p. 5-16, 2008.

MACHADO, S. A.; BAILEY, R. L.; BASSO, S. F.; BEVILACQUA JR, V. G. Análise do comportamento da relação hipsométrica com respeito à idade para plantações de Pinus elliottii no Paraná. Cerne, Lavras, v. 1, n. 1, p. 5-12, 1994.

MACHADO, S. A.; BARROS, D. A. de; SCOLFORO, J. R. S.; ACERBI JÚNIOR, F. W. The effects of successive thinnings on the hypsometric function for Pinus oocarpa stands. Floresta, v. 41, n. 2, p. 397-406, 2011

NICOLETTI, M. F.; SOUZA, K.; SILVESTRE, R.; FRANÇA, M. C.; ROLIM, F. A. Relação Hipsométrica para Pinus taeda L. em Diferentes Fases do Ciclo de Corte. Floresta e Ambiente, Seropédica, v. 23, n. 1, p. 80-89, 2016.

OLIVEIRA, L. T.; FERREIRA, M. Z.; CARVALHO, L. M. T.; FERRAZ FILHO, A. C.; OLIVEIRA, T. C. A.; SILVEIRA, E. M. O.; ACERBI JUNIOR, F. W. Determinação do volume de madeira em povoamento de eucalipto por escâner a laser aerotransportado. Pesquisa Agropecuária Brasileira, Brasília, v. 49, n. 9, p. 692699, 2014

PELISSARI, A. L.; DAVID, H. C.; PÉLlICO NETTO, S.; CALDEIRA, S. F.; FIGUEIREDO FILHO, A.; MARINHESKI FILHO, A. Effect of systematic sampling intensity in the hypsometric relationship of teak stands. Revista Brasileira de Biometria, Lavras, v. 34, n. 1, p. 23-32, 2016.

RETSLAFF, F. A. S.; FIGUEIREDO FILHO, A.; DIAS, A. N.; BERNETT, L. G.; FIGURA, M. A. Curvas de sítio e relações hipsométricas para Eucalyptus grandis na região dos Campos Gerais, Paraná. Cerne, Lavras, v. 21, n. 2, p. 219-225, 2015.

RIBEIRO, A.; FERRAZ FILHO, A. C.; MELlO, J. M.; FERREIRA, M. Z.; LISBOA, P. M. M.; SCOLFORO, J. R. S. Estratégias e metodologias de ajuste de modelos hipsométricos em plantios de Eucalyptus sp. Cerne, Lavras, v. 16, n. 1, p. 22-31, 2010.

SCHUMACHER, F. X.; HALL, F. S. Logarithmic expression of timber-tree volume. Journal of Agricultural Research, n. 47, p. 719-734, 1933.

SILVA, G. F.; XAVIER, A. C.; RODRIGUES, F. L.; PETERNELLI, L. A. Análise da influência de diferentes tamanhos e composições de amostras no ajuste de uma relação hipsométrica para Eucalyptus grandis. Árvore, Viçosa, v. 31, n. 4, p. 685-694, 2007.

SILVA, G. F.; OLIVEIRA, O. M.; SOUZA, C. A. M.; SOARES, C. P. B.; LEMOS, R. Influência de diferentes fontes de erro sobre as medições de alturas de árvores. Cerne, Lavras, v. 18, n. 3, p. 397-405, 2012.

SOARES, T. S.; SCOLFORO, J. R. S.; FERREIRA, S. O. Uso de diferentes alternativas para viabilizar a relação hipsométrica no povoamento florestal. Árvore, Viçosa, v. 28, n. 6, p. 845-854, 2004.

STOFFELS, A.; SOEST, J. VAN. The main problems in sample plots. Ned Bosbouwtijdschr, v. 25, p. 190-199, 1953.

THIERSCH, C. R.; ANDRADE, M. G.; MOREIRA, M. F. B.; LOIBEL, S. Estimativa da relação hipsométrica em clones de Eucalyptus sp. com o modelo de Curtis ajustado por métodos bayesianos empíricos. Árvore, Viçosa, v. 37, n. 1, p. 1-8, 2013. 